\section{Turmoil for medical doctors in Greece}

Sir:

Things were far from calm in the Greek medical community in the last months of 2009. The reason for this turmoil was a 3-month delay in the overtime payments of medical doctors working in hospitals throughout the country. Many doctors went on strike, which may seem like an extreme measure to some other citizens of the European Union (EU), but in Greece it is considered 'common policy'.

The general feeling has been that healthcare funding in Greece is less than adequate, with most hospitals being under-staffed and under-equipped, and Greek medical doctors cannot help but feel under-paid in comparison both with other professionals in Greece and with medical doctors in most other EU countries. A $20 \%$ rise in salaries in January 2009 did not hide the fact that Greek medical doctors' salaries are significantly below the EU average, while the cost of living in Greece is relatively high, and many doctors depend on their overtime payments to cover their basic expenses. Trainees, being the lowest-paid doctors, yet old enough to have their own families, have struggled the most.

In the psychiatric hospital of Attica, the largest psychiatric facility in Greece, with more than 100 psychiatrists and trainees, overtime was unpaid for 5 months, and doctors felt compelled to apply extreme measures, like closing the gates of the hospital. In mid-December there was finally some financial compensation, but the question had already been raised by some: are we stigmatised like our patients? The answer to that question requires much debate.

Problems concerning medical doctors in Greece seem to continue. There is an over-supply of medical professionals in Greece and an extremely long waiting-list for doctors to begin specialisation, both caused by an overproduction of medical graduates over the past 15 years. The future does not seem bright and sometimes loving what you do is the only thing keeping you going. Fortunately, it is enough, isn't it?

\section{Rafail Psaras \\ President of the Hellenic Association of Psychiatric Trainees;} Psychiatric Hospital of Attica, Athens, Greece, email rafailpsaras@yahoo.gr

\section{Clinical Assessment of Skills and Competencies examination}

\footnotetext{
C. In October 2009, we had the pleasure of - visiting the Clinical Assessment of Skills and Competencies (CASC) examination conducted by the Royal College of Psychiatrists in Sheffield. We were impressed by what we observed and left with the conviction that we in the USA have much to learn from the Royal College.
}

Both of us have participated extensively in psychiatric education and in the corresponding assessment processes conducted in psychiatry by the American Board of Psychiatry and Neurology. We were struck by the remarkable quality of the content of the College examination, as well as the efficiency and economy of the design and implementation. Each candidate performed an assigned clinical task in interaction with a simulator in each of 16 stations. The atmosphere was one of cheerful hard work and cooperation, with a shared sense of the importance of the assessments.

Each of the case vignettes was specifically constructed to assess core psychiatric clinical skills and we were impressed by the consistently clear internal validity. The four sets of linked simulations were especially creative and true to life for a psychiatrist practising in the community; they assessed skills in interviewing, diagnosis, psychoeducation and treatment, with a breadth and depth we have not seen before. Dr Anthony Bateman and his team of writers have done a superb job in this area. As participants in exam-writing teams for the American Board, we felt both envious and inspired.

We also noted the clarity of the roles of examiners and simulators, and the careful delineation of criteria for evaluating the candidates. Each examiner would observe the performance of a candidate in a station without interacting with him or her. Each simulator would interact with the candidate and simulate a patient or a family member or a consultant, without evaluating the candidate; those who took on the former roles were professional actors with long experience of simulations, while experienced psychiatrists simulated consultants (but, again, did not participate in evaluating the candidates). Examiners provided the simulators with feedback concerning their performance in the roles, but they did not discuss candidate performance with the simulators.

The grading process is described on the College website, but as outside observers we were impressed with the description of specific skills and competencies to be tested in each station, as well as the written criteria for the evaluation of performance. We found these descriptions to be specific, articulate and readily applicable, and we understood that they were written and rewritten in a well-organised process. The final phase in criterion development consisted of a 'calibration' conference at the beginning of the CASC day. In this discussion, examiners from each station addressed each other's questions and worked out the specific elements of a candidate's performance that would constitute a pass.

The CASC is an appropriately challenging and comprehensive evaluation process, which has required and benefited from a tremendous amount of effort and expertise on the part of the Royal College.

\section{Joan M. Anzia ${ }^{1}$ MD and David J. Lynn² MD}

'Associate Professor of Psychiatry, Vice Chair for Education, Department of Psychiatry and Behavioral Sciences, Northwestern University Feinberg School of Medicine, Chicago, Illinois, USA, email janzia@nmh.org 2Professor of Psychiatry, Loyola University Chicago Stritch School of Medicine, Maywood, Illinois, USA 